\title{
Fabry-Perot cavity based on polymer FBG as refractive index sensor
}

\author{
Miguel F.S. Ferreira ${ }^{a, *}$, Gabriela Statkiewicz-Barabach ${ }^{b}$, Dominik Kowal ${ }^{b}$, Pawel Mergo ${ }^{c}$, \\ Waclaw Urbanczyk ${ }^{\mathrm{b}}$, Orlando Frazão ${ }^{\mathrm{a}}$ \\ a INESC TEC and Department of Physics and Astronomy, Faculty of Sciences of University of Porto, Rua do Campo Alegre 687, 4169-007 Porto, Portugal \\ b Wroclaw University of Science and Technology, Faculty of Fundamental Problems of Technology, Wybrzeze Wyspianskiego 27, 50-370 Wroclaw, Poland \\ c Maria Curie-Sklodowska University, Laboratory of Optical Fibre Technology, Pl. M. Curie-Sklodowskiej 3, 20-031 Lublin, Poland
}

\section{A R T I C L E I N F O}

\section{Keywords:}

Polymer optical fibers

Fiber Bragg grating

Fabry-Perot interferometer

Refractive index sensor

\begin{abstract}
A B S T R A C T
The use of a polymer fiber as a refractive index sensor is proposed. A fiber Bragg grating is inscribed near the fiber tip and the fiber is cut shorter thus creating a Fabry-Perot cavity. The reflections between the fiber Bragg grating and the fiber end-face create a Fabry-Perot interferometer. The sensor was characterized to refractive index changes at constant temperature and to temperature at constant refractive index using a fast Fourier transform analysis of the interference signal. The sensor revealed a sensitivity of $-1.94 \quad \mathrm{RIU}^{-1}$ with a resolution of $1 \times 10^{-3} \mathrm{RIU}$ and low sensitivity to temperature, with a cross sensitivity to temperature of $3.6 \times 10^{-4} \mathrm{RIU} /{ }^{\circ} \mathrm{C}$.
\end{abstract}

\section{Introduction}

Polymer optical fibers (POFs) have been used for as long as conventional silica fibers. These fibers were overshadowed by their high propagation losses but present several advantages over silica fibers which make them interesting for sensor fabrication, thus the interest to study POFs for parameter sensing, like strain, temperature and refractive index has been increasing [1-4].

The measurement of the refractive index of liquid substances is important for several applications in medicine, biology and chemistry. Abbe refractometers are used to measure refractive index through the detection of the critical angle [5]. These refractometers are often large and not very practical for medical and biological applications as they require certain measurement conditions like the use and disposal of part of the sample. Fiber optic based sensors proved to be an alternative solution due to their small size, high sensitivity and other characteristics like biocompatibility. Most often their operation principle is based on the analysis of changes in the spectrum caused by a specific parameter change. For measuring refractive index the use of birefringent fibers [6-8], fiber gratings [6,7,9-12], optical fiber coatings $[13,14]$ and fiber interferometers $[6,8,9,15]$ have been already presented in literature.

Among different fiber refractometer concepts, the surface plasmon resonance (SPR) based devices allow for achieving particularly high measurement resolution, reaching $10^{-7}$ RIU [14]. Recently, it has been demonstrated [15] that by using micromachined two Fabry-Perot cavities at the tip of the fiber similar resolution can be achieved, however, manufacturing of such a sensor required multistep, complex procedure.

In spite of lower measurement resolution, fiber Bragg gratings (FBGs) [16] offer several advantages in refractive index sensing. Small sizes as well as the possibility of reflection interrogation and wavelength multiplexing are some of them. It has been shown [11] that FBGs written in suspended core fibers allow for measuring the refractive index change as small as $3 \times 10^{-5} \mathrm{RIU}$. Lower resolution of $3 \times 10^{-3} \mathrm{RIU}$ has been achieved by using an FBG written in H-shaped fiber [7], however, this solution made it easier for accessing the evanescent optical field and thus allowed for much faster measurements. Similar resolution of $5.5 \times 10^{-3} \mathrm{RIU}$ and possibility of improvement up to $5 \times 10^{-5}$ RIU have been reported by using FBGs written in exposed-core microstructured optical fibers [12]. On the other hand it is convenient to combine the FBG with a fiber tip where light reflects many times between the grating and the fiber end-face, thus creating Fabry-Perot cavity. The visibility of fringe pattern depends on the Fresnel reflection at the fiber end-face, therefore by dipping the tip of the fiber in a liquid one can measure its refractive index. This kind of sensor is easy to fabricate and allows for fast and simple operation. By now, refractometers of this design were reported for silica fibers $[6,9]$ yielding a sensitivity ranging from $-1.06 \quad \mathrm{RIU}^{-1}$ to $-3 \quad \mathrm{RIU}^{-1}$ and the resolution of $10^{-3}$ RIU.

The first FBGs in POFs were fabricated in late 1990s [17] and since then such structures have been gaining increasing attention $[18,19]$. In this paper, the use of Fabry-Perot interferometer formed at the tip of the polymer fiber for refractive index measurements was investigated.

\footnotetext{
* Corresponding author.

E-mail address: miguel.fs.ferreira93@gmail.com (M.F.S. Ferreira).
} 


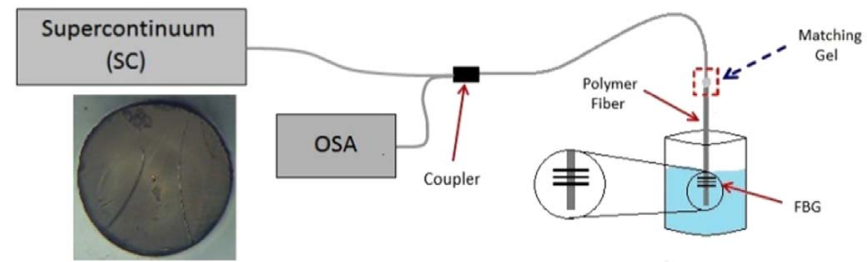

Fig. 1. Experimental setup for characterization of the Fabry-Perot interferometer based sensor. On the inset a cross section of the POF is shown.

We demonstrate for the first time the FP cavity in POF created between a fiber Bragg grating and the fiber end-face. The FBG is imprinted in the fiber using the phase mask method [17-19] near the tip and then the tip is cut shorter to create the cavity. The measurement method proposed was similar to previous studies in silica fibers [6,9]. However, using POFs for sensors fabrication opens new application opportunities in medicine and biology due to better biocompatibility of polymer fibers. Moreover, the use of polymer fibers eliminates a practical limitation of sensors based on silica fibers related to fragility of the fiber tip which might get damaged during sensing procedure.

\section{Experimental setup}

The experimental setup is shown in Fig. 1. A supercontinuum was required to cover the full reflection spectrum of the interferometer around the window of $1.3 \mu \mathrm{m}$. In the following experiments a supercontinuum (SC) source was used (NKT Photonics - SuperK Versa) and the reflected signal was observed on the optical spectrum analyzer (Yokogawa AQ6370D). For the Bragg grating inscription a step-index POF was used, fabricated at Maria Curie-Sklodowska University in Lublin, Poland. It was composed of poly(methyl methacrylate) (PMMA) in the cladding and a copolymer of PMMA and polystyrene (PS) in the core ( $5 \mathrm{w} / \mathrm{w} \%)$. The outer diameter of the fiber was $268 \mu \mathrm{m}$ and the diameter of the core was $3.8 \mu \mathrm{m}$. The connection between the silica fiber and the POF required alignment and matching gel in order to couple the light into the core and avoid creating a cavity between both fibers.

For inscribing the FBG in POF, a UV laser beam (CW He-Cd laser Kimmon IK3301R-G, $\lambda=325 \mathrm{~nm}$ ) was focused on the fiber through a cylindrical lens and a phase mask. The inscribed grating had a length of approximately $1.4 \mathrm{~mm}$. In the reflection spectrum of the fabricated FBG two peaks were visible. The peak splitting appears most likely because the POF has not been annealed prior to the grating inscription. If the fiber is irradiated for some time, the spectrum may get distorted [19]. This effect originates from temperature increase occurring in POFs when exposed to the UV which results in random grating shrinkage due to stress relaxation and affects the reflection spectrum.

After writing an FBG the fiber was cleaved in close distance to the grating. As the light was reflected back and forward between the FBG and the end of the fiber, a cavity was formed and a fringe pattern appeared on the reflection spectrum of the FBG. A spectral separation of adjacent interference fringes observed in the reflected spectrum can be expressed as $\Delta \lambda=\lambda_{0} / 2 \mathrm{nl}$, where $\mathrm{n}$ is the effective refractive index of the core, $l$ is the length of the cavity and $\lambda_{0}$ is the central wavelength of the fringe pattern. Fig. 2 shows the fringe pattern for different cavity lengths (the fiber was cut several times). During each cutting procedure the fiber tip was annealed in the cutting board which shifted the spectrum (the temperature of the cutting board was about $73^{\circ} \mathrm{C}$ ). In its final shape the spectrum consisted of the fringes of the periodicity $\Delta \lambda$ equal to $0.51 \mathrm{~nm}$ which corresponds to the cavity length of $1.1 \mathrm{~mm}$ (Fig. 2d). The fringe visibility was equal to $68 \%$ which means that the optical power reflected by the FBG and the end-face of POF did not differ significantly. Therefore, reflectance of the grating was close to the reflectance of the end-face that, due to cut imperfections, was smaller than $4 \%$, which is due to long irradiation time used in fabrication of the

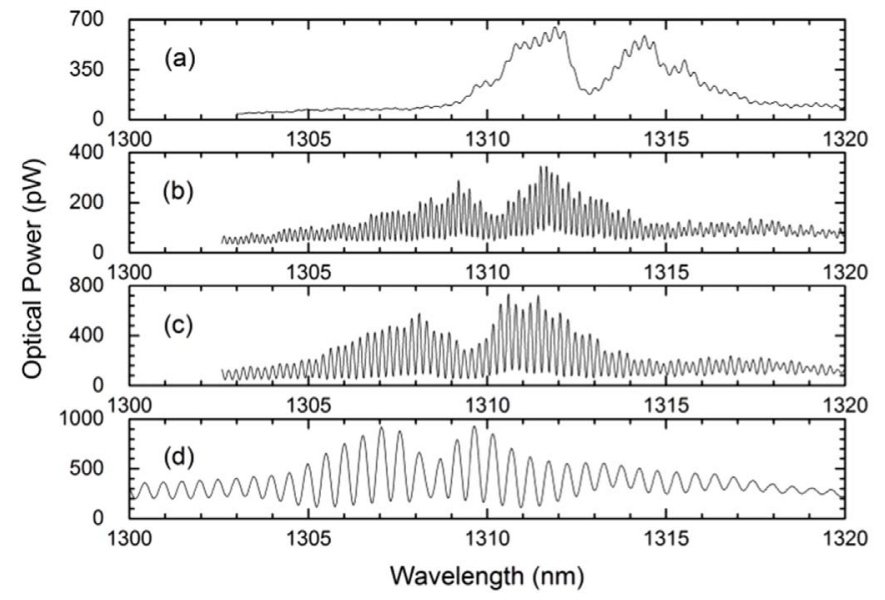

Fig. 2. (a) Reflection spectrum of the FBG before making a cavity. (b) Reflection spectrum with a cavity length equal to $3.6 \mathrm{~mm}$, (c) $2.8 \mathrm{~mm}$, (d) $1.1 \mathrm{~mm}$ recorded in air.

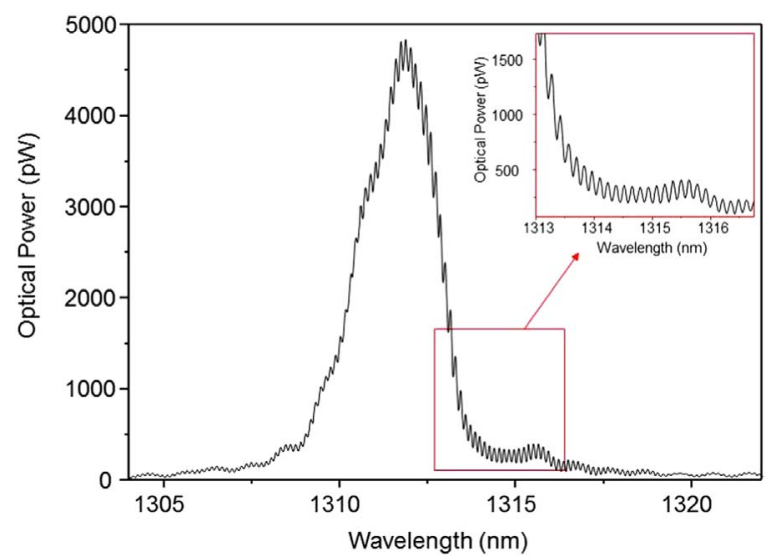

Fig. 3. Reflection spectrum of a Fabry-Perot interferometer formed by an FBG and fiber end-face recorded in air. The FBG exhibits higher reflectance than the one in Fig. 2.

FBG. For comparison, another grating with much higher reflectance was fabricated and a FP cavity was formed again. It can be seen in Fig. 3 that the fringe visibility in this case was much lower due to a much stronger reflectance in the grating than in the end-face of the fiber which leads to a weak interference from both reflections.

\section{Results}

Glycerin solutions in water of different concentrations were prepared to test the performance of the sensor. Using an Abbe refractometer, the solutions were measured ranging from 1.33800 to 1.47650 RIU. Around $1.5 \mathrm{~cm}$ of tip of the fiber were dipped into every liquid and the spectrum acquisition was done right after during a $15 \mathrm{~s}$ period. Therefore, short time required for obtaining the result was one of the advantages of the presented setup. Dipping would alter the reflection in the end-face of the fiber due to presence of a different refractive index. With a different reflection, the interference pattern changes in amplitude since the difference between both reflectances is different.

In Fig. 4 it is visible that the average power and the contrast of interference fringes were dropping with the increase of the refractive index. The spectra were analyzed using a fast Fourier transform (FFT) in order to obtain the amplitude of the frequency of the fringes (Fig. 5). In order to avoid problems with the power source or any other instability during the measurements, the amplitude was normalized with respect to the amplitude of the fringes in air, registered prior to each measurement of liquid with different refractive index. For the normalized amplitude a linear behavior was observed against refractive 


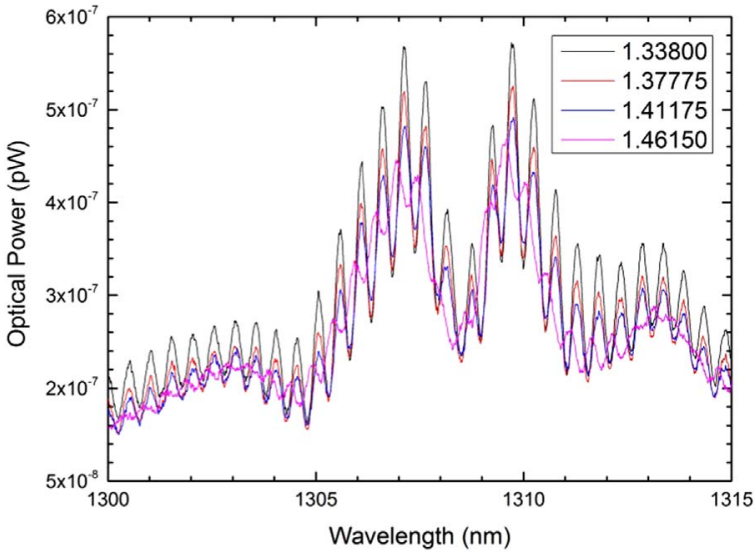

Fig. 4. Reflection spectra of a FP cavity for different refractive indices, the contrast of interference fringes drops with increasing refractive index.

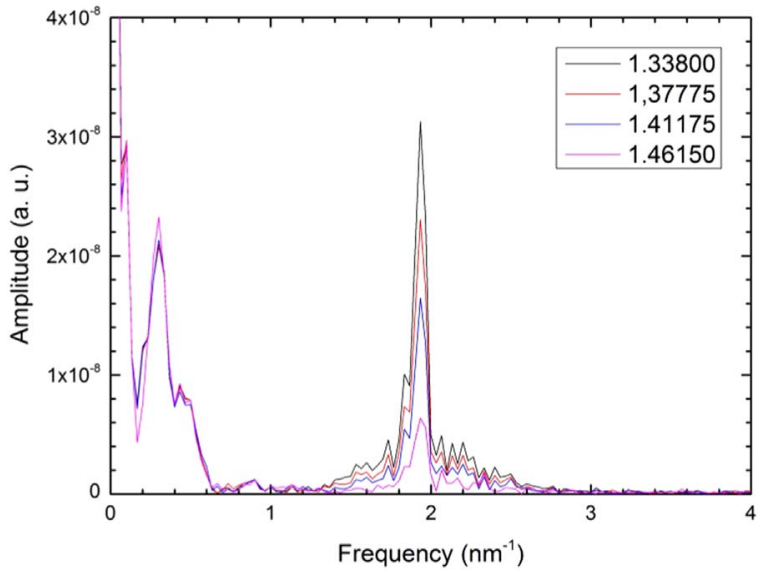

Fig. 5. FFTs of the reflection spectra for 4 liquids with refractive indices of 1.33800 , $1.37775,1.41175$ and 1.46150 .

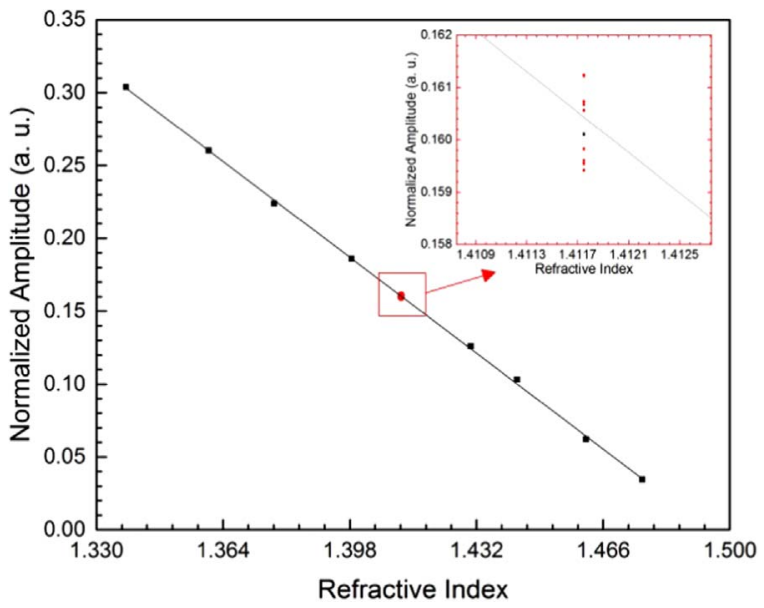

Fig. 6. Dependence of normalized amplitude of the frequency of the interference fringes upon refractive index changes.

index changes (Fig. 6). A sensitivity of $-1.94 \mathrm{RIU}^{-1}$ was obtained with this sensor after temperature characterization. This sensitivity is complied with previous preliminary data with oil based samples prior to the temperature characterization displaying no eventual changes with annealing [20]. Ten measurements of the same liquid were done and the scattering of the results was $0.0018 \mathrm{a}$. u. which is shown on the inset of the Fig. 6 . Therefore, the estimated measurement resolution of the sensor is equal to $1 \times 10^{-3} \mathrm{RIU}$.

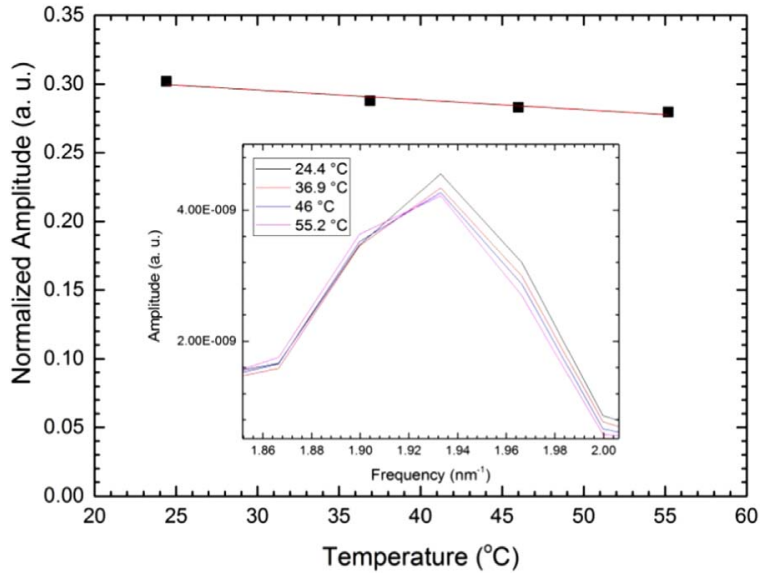

Fig. 7. FFTs of the spectra at different temperatures. The normalized amplitude of the peak frequency of the fringes drops with the increase of the temperature.

Moreover, the sensor was tested for the temperature response in the range $24.4-55.2{ }^{\circ} \mathrm{C}$. The FFT was used to analyze the reflection spectra (Fig. 7), achieving a low sensitivity of $-7 \times 10^{-4 \circ} \mathrm{C}^{-1}$. This behavior implies that the difference between the refractive indices of the polymer fiber and the water decreases with the increase of temperature. Since both values drop with temperature in the examined range $[21,22]$, the refractive index for polymer fiber drops faster than the one for water. It is important to calculate the ratio between the two sensitivities, to temperature and to refractive index of the liquid, also known as cross sensitivity. A cross sensitivity of $3.6 \times 10^{-4} \mathrm{RIU} /{ }^{\circ} \mathrm{C}$ was attained, thus, for small temperature deviations, the sensor can read up to 3 decimal cases.

\section{Conclusion}

In this paper, the study of POF as a liquid refractive index sensor is shown. The analysis was made using a fast Fourier transform and observing the amplitude of the peak frequency. The sensor had a linear response with a resolution of $1 \times 10^{-3} \mathrm{RIU}$. The results were reproducible and a refractive index sensitivity of $-1.94 \mathrm{RIU}^{-1}$ was obtained in a range from 1.33800 to 1.47650 . It is worth to emphasize that presented setup allowed for making quick measurements, depending only on the time for a spectrum acquisition. Moreover, the biocompatibility and sturdiness of POFs make the sensor have a broader application and more resistance to damage than silica fibers.

\section{Acknowledgement}

This work was supported by the grant for bilateral scientific cooperation between Poland and Portugal and in part by Fundação para a Ciência e Tecnologia (Portuguese Foundation for Science and Technology), in part by the European Regional Development Fund (ERDF) through the COMPETE Programme (Operational Programme for Competitiveness) within Project FCOMP-01-0124-FEDER-037281.

\section{References}

[1] X.F. Chen, C. Zhang, D.J. Webb, G.-D. Peng, K. Kalli, Bragg grating in polymer optical fibre for strain, bend and temperature sensing, Meas. Sci. Technol. 21 (2010) 94005. http://dx.doi.org/10.1088/0957-0233/21/9/094005.

[2] G. Statkiewicz-Barabach, D. Kowal, M.K. Szczurowski, P. Mergo, W. Urbanczyk, Hydrostatic pressure and strain sensitivity of long period grating fabricated in polymer microstructured fiber, IEEE Photonics Technol. Lett. 25 (2013) 496-499.

[3] J. Jensen, P. Hoiby, G. Emiliyanov, O. Bang, L. Pedersen, A. Bjarklev, Selective detection of antibodies in microstructured polymer optical fibers, Opt. Express 13 (2005) 5883-5889. http://dx.doi.org/10.1364/OPEX.13.005883.

[4] V. Duong, Ta, R. Chen, L. Ma, Y. Jun Ying, H. Dong Sun, Whispering gallery mode microlasers and refractive index sensing based on single polymer fiber, Laser Photon. Rev. 7 (2013) 133-139. http://dx.doi.org/10.1002/lpor.201200074.

[5] G.H. Meeten, A.N. North, Refractive index measurement of absorbing and turbid 
fluids by reflection near the critical angle, Meas. Sci. Technol. 6 (1995) 214-221.

[6] C. Gouveia, P.A.S. Jorge, J.M. Baptista, Fabry - Pérot cavity based on a highbirefringent fiber Bragg grating for refractive index and temperature measurement, IEEE Sens. J. 12 (2012) 17-21.

[7] O. Frazão, T. Martynkien, J.M. Baptista, J.L. Santos, W. Urbanczyk, J. Wojcik, Optical refractometer based on a birefringent Bragg grating written in an $\mathrm{H}$-shaped fiber, Opt. Lett. 34 (2009) 76-78.

[8] O. Frazão, P. Jorge, J.M. Baptista, J.L. Santos, Optical refractometer based on a HiBi D-type fiber loop mirror, in: IEEE Sensors, Lecce, 2008, pp. 957-960. 〈http:// dx.doi.org/1-4244-2581-5/08/\$20.00@2008IEEE

[9] S.F.O. Silva, O. Frazão, P. Caldas, J.L. Santos, F.M. Araújo, L.A. Ferreira, Optical fiber refractometer based on a Fabry- Pérot interferometer, Opt. Eng. 47 (2008) 54403-54403-5. http://dx.doi.org/10.1117/1.2931527.

[10] V. Bhatia, Applications of long-period gratings to single and multi-parameter sensing, Opt. Express 4 (1999) 457-466. http://dx.doi.org/10.1364/OE.4.000457.

[11] M. Châu, P. Huy, G. Laffont, V. Dewynter, P. Ferdinand, P. Roy, J. Auguste, D. Pagnoux, W. Blanc, B. Dussardier, Three-hole microstructured optical fiber for efficient fiber Bragg grating refractometer, Opt. Lett. 32 (2007) 2390-2392.

[12] S.C. Warren-Smith, T.M. Monro, Exposed core microstructured optical fiber Bragg gratings: refractive index sensing, Opt. Express 22 (2014) 1480-1489. http:// dx.doi.org/10.1364/OE.22.001480.

[13] C.R. Zamarreño, P. Sanchez, M. Hernaez, I. Del Villar, C. Fernandez-Valdivielso, I.R. Matias, F.J. Arregui, Dual-peak resonance-based optical fiber refractometers, IEEE Photonics Technol. Lett. 22 (2010) 1778-1780.

[14] D. Monzón-Hernández, J. Villatoro, High-resolution refractive index sensing by means of a multiple-peak surface plasmon resonance optical fiber sensor, Sens. Actuators B Chem. 115 (2006) 227-231. http://dx.doi.org/10.1016/ j.snb.2005.09.006.

[15] S. Pevec, D. Donlagic, High resolution, all-fiber, micro-machined sensor for simultaneous measurement of refractive index and temperature, Opt. Express 22 (2014) 16241-16253. http://dx.doi.org/10.1364/OE.22.016241.

[16] K.O. Hill, G. Meltz, Fiber Bragg grating technology fundamentals and overview, J. Light. Technol. 15 (1997) 1263-1276.

[17] Z. Xiong, G.D. Peng, B. Wu, P.L. Chu, Highly tunable Bragg gratings in single-mode polymer optical fibers, IEEE Photonics Technol. Lett. 11 (1999) 352-354. http:// dx.doi.org/10.1109/68.748232.

[18] G. Statkiewicz-Barabach, K. Tarnowski, D. Kowal, P. Mergo, W. Urbanczyk, Fabrication of multiple Bragg gratings in microstructured polymer fibers using a phase mask with several diffraction orders, Opt. Express 21 (2013) 8521-8534. http://dx.doi.org/10.1364/OE.21.008521.

[19] G. Statkiewicz-Barabach, D. Kowal, P. Mergo, W. Urbanczyk, Comparison of growth dynamics and temporal stability of Bragg gratings written in polymer fibers of different types, J. Opt. 17 (2015) 85606. http://dx.doi.org/10.1088/2040-8978/ $17 / 8 / 085606$.

[20] A. Pospori, C.A.F. Marques, M.G. Zubel, D. Sáez-Rodríguez, K. Nielsen, O. Bang, D. J. Webb, Annealing effects on strain and stress sensitivity of polymer optical fibre based sensors, in: Proceedings SPIE, 2016, p. 98860V-98860V-7. 〈http://dx.doi. org/10.1117/12.2227473>.

[21] P. Michel, J. Dugas, J.M. Cariou, L. Martin, Thermal variations of refractive index of PMMA, polystyrene, and poly ( 4-methyl-1-pentene), J. Macromol. Sci. Part B Phys. 25 (1986) 379-394.

[22] T. Wei, Y. Han, Y. Li, H. Tsai, H. Xiao, Temperature-insensitive miniaturized fiber inline Fabry-Perot interferometer for highly sensitive refractive index measurement, Opt. Express 16 (2008) 5764-5769. 